\title{
Rapid detection of influenza A (H1N1) virus by conductive polymer- based nanoparticle via optical response to virus-specific binding
}

\author{
Geunseon Park ${ }^{1, \S}$, Hyun-Ouk Kim ${ }^{2,3,}$, Jong-Woo Lim ${ }^{1}$, Chaewon Park ${ }^{1}$, Minjoo Yeom ${ }^{4}$, Daesub Song ${ }^{4}(\bowtie)$, and \\ Seungjoo $\operatorname{Haam}^{1}(\triangle)$ \\ ${ }^{1}$ Department of Chemical \& Biomolecular Engineering, Yonsei University, Seoul 03722, Republic of Korea \\ ${ }^{2}$ Division of Chemical Engineering and Bioengineering College of Art, Culture and Engineering, Kangwon National University, Chuncheon-si, \\ Gangwon-do 24341, Republic of Korea \\ ${ }^{3}$ Biohealth-machinery Convergence Engineering, Kangwon National University, Chuncheon, Gangwon-do 24341, Republic of Korea \\ ${ }^{4}$ College of Pharmacy, Korea University, Sejong 30019, Republic of Korea \\ ${ }^{\S}$ Geunseon Park and Hyun-Ouk Kim contributed equally to this work.
}

(C) Tsinghua University Press and Springer-Verlag GmbH Germany, part of Springer Nature 2021

Received: 14 May 2021 / Revised: 22 July 2021 / Accepted: 25 July 2021

\section{ABSTRACT}

A recurrent pandemic with unpredictable viral nature has implied the need for a rapid diagnostic technology to facilitate timely and appropriate countermeasures against viral infections. In this study, conductive polymer-based nanoparticles have been developed as a tool for rapid diagnosis of influenza $A(\mathrm{H} 1 \mathrm{~N} 1)$ virus. The distinctive property of a conductive polymer that transduces stimulus to respond, enabled immediate optical signal processing for the specific recognition of $\mathrm{H} 1 \mathrm{~N} 1 \mathrm{virus}$. Conductive poly(aniline-co-pyrrole)-encapsulated polymeric vesicles, functionalized with peptides, were fabricated for the specific recognition of $\mathrm{H} 1 \mathrm{~N} 1$ virus. The low solubility of conductive polymers was successfully improved by employing vesicles consisting of amphiphilic copolymers, facilitating the viral titer-dependent production of the optical response. The optical response of the detection system to the binding event with $\mathrm{H} 1 \mathrm{~N} 1$, a mechanical stimulation, was extensively analyzed and provided concordant information on viral titers of $\mathrm{H} 1 \mathrm{~N} 1$ virus in $15 \mathrm{~min}$. The specificity toward the H1N1 virus was experimentally demonstrated via a negative optical response against the control group, H3N2. Therefore, the designed system that transduces the optical response to the target-specific binding can be a rapid tool for the diagnosis of $\mathrm{H} 1 \mathrm{~N} 1$.

\section{KEYWORDS}

influenza $\mathrm{A}(\mathrm{H} 1 \mathrm{~N} 1)$ virus, conductive polymer, optical property, rapid detection

\section{Introduction}

Public health has faced a serious challenge by the recent coronavirus disease (COVID-19) pandemic caused by infections with severe acute respiratory syndrome coronavirus 2 (SARS-CoV2 ). The number of confirmed cases is more than 85 million globally, and over millions of death cases have been reported to the World Health Organization (WHO) as of January 6, 2021. The present viral outbreak is not the only one that has threatened public health. There have been incidences of recurrent viral infections, such as dengue, Zika, SARS, Middle East respiratory syndrome (MERS), and influenza viral infections worldwide [1-3]. The history of recurrent viral outbreaks implies that evolving viruses with an unpredictable nature may continuously arise. Moreover, various types of viruses frequently mutate their genomic information, which increases the uncertainty in the prevention of the spread of infectious diseases [4-7]. WHO underlined the need to improve emergency preparedness due to the fatality of outbreaks in urban environments with increased population mobility. Thus, countermeasures against the spread of viral infections in an early stage are very important, and the development of rapid diagnostics technologies is highly required $[8,9]$.
Conventional diagnostic techniques such as enzyme-linked immunosorbent assay (ELISA) and real-time quantitative polymerase chain reaction (qPCR) have been used to detect pathogens. ELISA can be utilized to detect a wide range of pathogens; however, it requires time-consuming multiple steps from preparation to detection [10-15]. Although real-time qPCR analysis is regarded as the gold standard due to its high sensitivity and accuracy, the accessibility of the technique is limited to test experts in the laboratory. Thus, a rapid and simple diagnostic test to address the early stage of viral infections is needed to assist in scenarios of urgency $[16,17]$.

To date, conductive polymers have been employed as simple and effective tools for analytical investigations because transitions in the charge distribution, orientation, and electronic structure of conductive polymers are immediately presented by significant changes in physicochemical properties [18-21]. The instantaneous transduction of stimuli into response enabled conductive polymers to be applied as a rapid detection assay that responded to the intermolecular interaction of the target and corresponding biorecognition elements, such as antibodies, enzymes, and proteins [22-24]. Polyaniline (PANI) is a conductive polymer that exhibits electrochemical stability, excellent biocompatibility, high 
conductivity, and electro-optical properties [25-27]. PANI is highly responsive to $\pi-\pi$ interactions induced by diverse external stimuli, such as light, chemical doping, $\mathrm{pH}$, redox, and binding effects. Among the physicochemical properties of PANI that transduce stimulus into detectable signals, an optical response (O.R.) can be an optimal candidate for biosensors because detection via an optical detector is simple and does not require expensive instrumentation, which meets crucial conditions in point-of-care diagnostics [28-34].

In this study, a simple and rapid assay for the detection of influenza A (H1N1) virus was designed with the resonance structure of a conductive polymer [poly(aniline-co-pyrrole)] encapsulated vesicle (CPV) (Fig. 1). CPV nanostructures with amphiphilic polymers overcome the poor water dispersion of conductive polymers and enable stable detection of aqueous target virus specimens $[35,36]$. CPVs expose distinctive absorbance spectrums responding to different distances between CPVs because the strength of $\pi-\pi$ interactions is dependent on the distance [37]. We combined the CPVs with a peptide having a specificity toward hemagglutinin, which is the target moiety of the influenza virus [38]. In the presence of $\mathrm{H} 1 \mathrm{~N} 1$ virus, the peptideconjugated CPV (PCPV) specifically binds to the virus, reducing the distance between the CPVs. The agglomeration of PCPV with selective recognition of the target virus functioned as a mechanical stimulation, shifting $\pi-\pi$ interactions, and triggered an optical response that facilitates the quantitative detection of $\mathrm{H} 1 \mathrm{~N} 1$ virus [39-41]. PCPV can execute user-friendly and rapid detection in 15 min via simple mixing with the specimen, corresponding with the diagnostic criteria to develop suitable sensing materials and strategies $[42,43]$. This sensing strategy puts weight on the optical properties of conductive polymers and provides a novel perspective on the development of biosensors that deviate from prevalent electrochemical sensors, contributing to advances in diagnostic procedures that could help control viral spread.

\section{Experimental section}

\subsection{Materials}

Triphosgene was purchased from Tokyo Chemical Industry Co., Ltd. $\alpha$-Methoxy- $\omega$-amino-poly(ethylene glycol) (mPEG- $\mathrm{NH}_{2}$ ), with a molecular weight of 2,000 g.mol ${ }^{-1}$, was purchased from Laysan Bio Inc. L-Phenylalanine (Phe), pyrrole, aniline, tetrahydrofuran (THF), N,N-dimethylformamide (DMF), dimethyl sulfoxide (DMSO), 1-ethyl-3-(3-dimethylamino-

(a) Conductive-polymer-based nanoparticle with specificity toward Influenza A virus

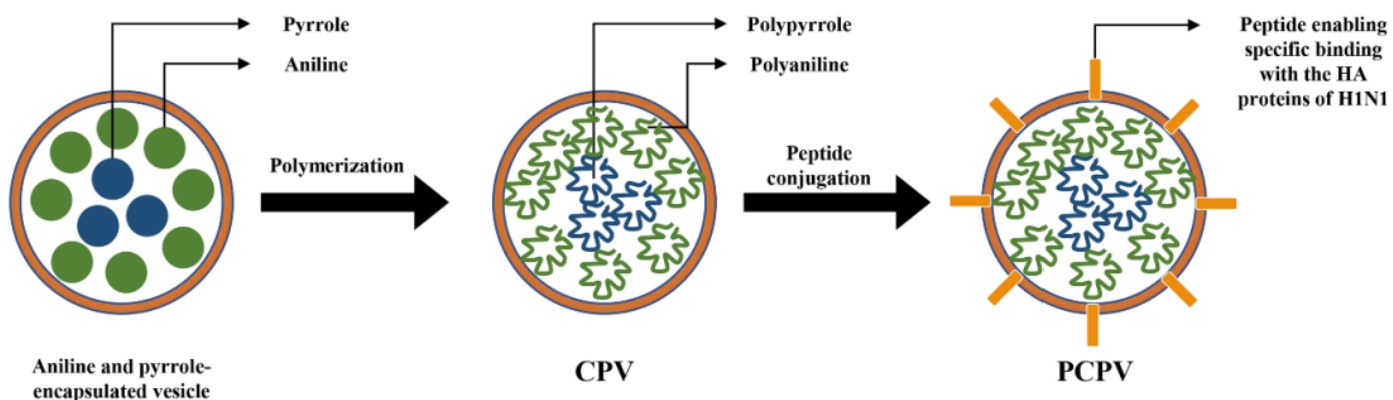

(b) Virus detection process
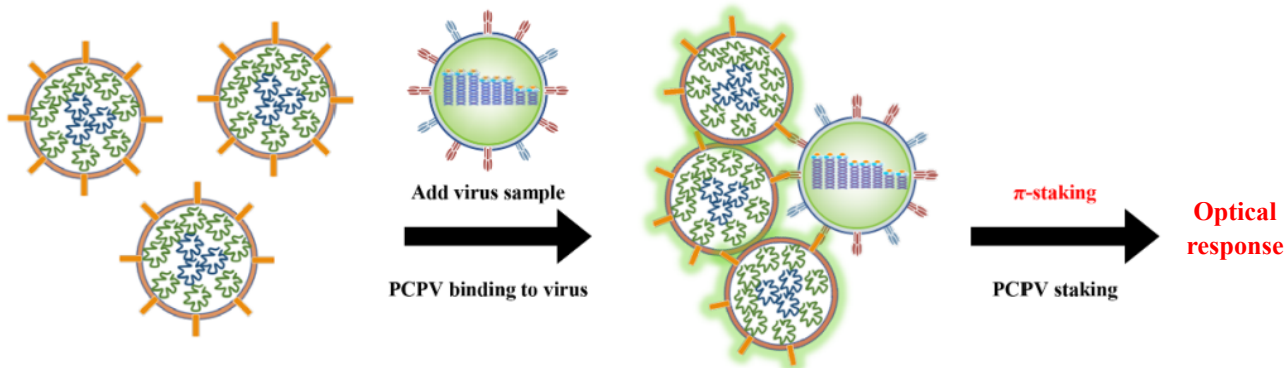

(c) Identification-driven workflow to quantify
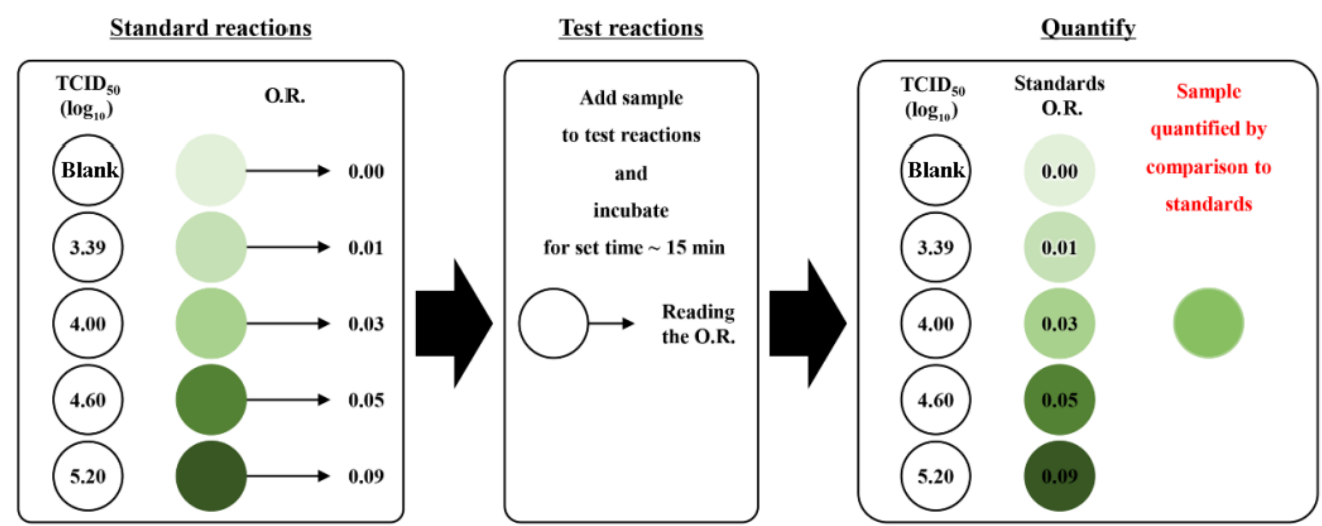

Figure 1 (a) Schematic illustration of preparation of conductive polymer-based nanoparticle with specificity toward H1N1 virus, and (b) virus detection process. (c) Identification-driven workflow to quantify H1N1 virus by PCPV. PCPV showed quantitative detection of H1N1 virus stocks within 15 min at room temperature. 
propyl)carbodiimide hydrochloride (EDC-HCl), and $\mathrm{N}$ hydroxysulfosuccinimide (Sulfo-NHS) were purchased from Sigma-Aldrich, MO. Diethyl ether, chloroform, n-hexane, and toluene were purchased from DUKSAN. Peptide for H1N1 virusspecific interaction (Fmoc-ARLSPTMVHPNGAQP- $\mathrm{NH}_{2}$ ) was conjugated from PEPTRON (Daejeon, Republic of Korea). All other chemicals and reagents were of analytical grade.

\subsection{Synthesis of methoxyl poly(ethylene glycol) block polyphenylalanine (mPEG-b-pPhe) and CM(Carboxy- methyl)-PEG-b-pPhe}

mPEG-b-pPhe and CM-PEG-b-pPhe were synthesized by ringopening polymerization of phenylalanine $\mathrm{N}$-carboxyanhydride (Phe-NCA), initiated by mPEG-NH $\mathrm{N}_{2}$ and CM-PEG-NH ${ }_{2}$, respectively (Fig. S1 in the Electronic Supplementary Material (ESM)). First, Phe-NCA was synthesized and later reacted with mPEG-NH ${ }_{2}$ to form mPEG-b-pPhe and CM-PEG-NH $\mathrm{N}_{2}$ to form CM-PEG-b-pPhe. Phenylalanine (3 g, $18.16 \mathrm{mmol}$ ) was dissolved in $100 \mathrm{~mL}$ of THF in a 3-neck round flask to synthesize phe-NCA. Triphosgene (2.8 g, $9.34 \mathrm{mmol})$ dissolved in $10 \mathrm{~mL}$ of THF was added dropwise to the above solution at a constant rate. The solution was allowed to react under stirring for $3 \mathrm{~h}$ under a nitrogen atmosphere at $50{ }^{\circ} \mathrm{C}$. The resultant product was filtered and precipitated in ice-cold hexane. The product was stored overnight at $-20^{\circ} \mathrm{C}$. Phe-NCA was filtered under a vacuum and washed with $\mathrm{n}$-hexane three times. To synthesize mPEG-b-pPhe

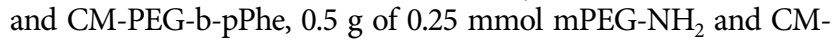
PEG-b-pPhe were dissolved in $5 \mathrm{~mL}$ of DMF in a 3-neck round bottom flask and $0.5 \mathrm{~g}, 3.03 \mathrm{mmol}$ solution of Phe-NCA, dissolved in $5 \mathrm{~mL}$ of DMF, was introduced, respectively. The solution was reacted for $24 \mathrm{~h}$ in a water bath while stirring at $37^{\circ} \mathrm{C}$. The resultant products were precipitated using a mixture of diethyl ether/DMF and filtered under a vacuum.

\subsection{Preparation of CPV}

CPV was synthesized via self-assembly of amphiphilic block copolymers, mPEG-b-pPhe, and CM-PEG-b-pPhe, which were synthesized in the same way. mPEG-b-pPhe $(2.5 \mathrm{mg})$ and CMPEG-b-pPhe $(2.5 \mathrm{mg})$ were dissolved in chloroform $(1 \mathrm{~mL})$, which is highly volatile, and the solution was poured into $10 \mathrm{~mL}$ of deionized water. The chloroform was evaporated while stirring for $24 \mathrm{~h}$. Aniline $(40 \mu \mathrm{L}, 0.45 \mathrm{mmol})$ and pyrrole $(3.5 \mu \mathrm{L}, 0.05 \mathrm{mmol})$ were added, in order, to the solution. The mixture was stirred for $30 \mathrm{~min}$ and ultrasonicated for $10 \mathrm{~min}$ to load aniline and pyrrole into the polymer vesicle, which self-assembled during the process. As a reducing agent, ammonium persulfate (APS) solution (1.1 M, $0.25 \mathrm{~mL}$ ) was added to the mixture. After $1 \mathrm{~min}$, the stirring process was stopped and the mixture was kept at $4{ }^{\circ} \mathrm{C}$ for $48 \mathrm{~h}$ to produce mature polyaniline and polypyrrole in the vesicles. The final product was purified using centrifugation.

\subsection{Characterization of the synthesized polymers and CPVs}

The chemical structures of Phe-NCA and the block copolymer were evaluated by Fourier transform infrared (FT-IR) spectroscopy (PerkinElmer, UATR Two), confirming the existence of the characteristic bands. The characteristic peaks of Phe-NCA in the FT-IR spectra are as follows: anhydride $\mathrm{C}=\mathrm{O}$ peaks at 1,854 and $1,760 \mathrm{~cm}^{-1}$. The characteristic peaks of mPEG-bpPhe and CM-PEG-b-pPhe in the FT-IR spectra are as follows: $\mathrm{N}-\mathrm{H}$ stretching vibration at $3,300 \mathrm{~cm}^{-1} ; \mathrm{C}=\mathrm{O}$ stretching vibration at $1,624 \mathrm{~cm}^{-1} ; \mathrm{N}-\mathrm{H}$ bending and $\mathrm{C}-\mathrm{N}$ stretching vibration (amide II) at $1,521 \mathrm{~cm}^{-1}$ (Fig. S2(a) in the ESM). In addition, the polymer dispensed in deuterated dimethyl sulfoxide- $\mathrm{d}_{6}\left(\right.$ DMSO- $\left.\mathrm{d}_{6}\right)$ was loaded onto a $400 \mathrm{MHz}{ }^{1} \mathrm{H}-\mathrm{NMR}$ spectrometer (Avance II, Bruker Biospin) to confirm the polymerization process. ${ }^{1} \mathrm{H}$ NMR (400 MHz, DMSO-d $\mathrm{d}_{6}$ ) $\delta: 9.08$ (-NH- of Phe-NCA), 7.16-7.28 (-CHof pPhe), $3.38\left(-\mathrm{OCH}_{3}-\right.$ of $\mathrm{mPEG}$ ) (Fig. S2(b) in the ESM). The distinctive optical properties of $\mathrm{CPV}$ were evaluated under various $\mathrm{pH}$ conditions with adjusted $\mathrm{pH}$ conditions with $1 \mathrm{M} \mathrm{HCl}$ or $1 \mathrm{M}$ $\mathrm{NaOH}$ using a multimode microplate reader (SpectraMax i3x, Molecular Devices).

\subsection{Conjugation of CPV and peptide having specific binding to hemagglutinin}

$\mathrm{CPV}$ and a peptide having specific binding to hemagglutinin were conjugated using EDC-NHS coupling. The carboxyl groups of CM-PEG-b-pPhe $\left(0.4 \mathrm{mg} \cdot \mathrm{mL}^{-1}\right)$ in the CPV $\left(0.5 \mathrm{mg} \cdot \mathrm{mL}^{-1}\right)$ were coupled to the amine groups of the peptide terminal via peptide bond formation. To facilitate the process sufficiently, excessive EDC and Sulfo-NHS in 10:1 and 20:1 molar ratios to CM-PEG-b$\mathrm{pPhe}$, respectively, were used. The mixture was stirred for $2 \mathrm{~h}$ at room temperature. The peptide $\left(1 \mathrm{mg} \cdot \mathrm{mL}^{-1}\right)$ in $20 \% \mathrm{DMSO}$ $(0.2 \mathrm{~mL})$ was added to $1 \mathrm{~mL}$ of CPVs and stirred for $4 \mathrm{~h}$. The product was purified using centrifugation. The conjugation of the peptide with CPV was confirmed using a $400 \mathrm{MHz}{ }^{1} \mathrm{H}-\mathrm{NMR}$ spectrometer (Avance II, Bruker Biospin). ${ }^{~} \mathrm{H}$ NMR (400 MHz, DMSO-d $\left.\mathrm{d}_{6}\right) \delta: 1.05\left(-\mathrm{CH}_{3}-\right.$ of peptide) (Fig. $\mathrm{S} 3$ in the ESM).

\subsection{Analysis of absorbance spectrum of the system and} calculation of optical response

The absorbance spectrum of CPV, CPV-liner, and PCPV was analyzed using a multimode microplate reader (SpectraMax i3x, Molecular Devices), in a range of wavelengths from 400 to 800 $\mathrm{nm}$. We adopted an optical response as a parameter of the relative deviation from the standard, and the parameter can be calculated by summation of the difference in absorbance intensity between the specimen $\left(A^{x}\right)$ and standard $\left(A^{\mathrm{STD}}\right)$ at 430 and $630 \mathrm{~nm}$, respectively (Eq. (1)). In a study to confirm the optical response against mechanical stimulation, the standard point of the absorbance intensity was at a concentration of $0.25 \mathrm{mg} \cdot \mathrm{mL}^{-1}$ for $\mathrm{CPV}$ and CPV-L and the same concentration of PCPV was the standard point in virus detection.

$$
\text { Optical response }(\text { O.R. })=\left(A_{430}^{x}-A_{430}^{\mathrm{STD}}\right)+\left(A_{630}^{\mathrm{STD}}-A_{630}^{x}\right)
$$

\subsection{Viruses}

Human influenza H1N1 (A/California/04/2009), canine influenza virus (CIV) H3N2 (A/canine/Korea/01/2007 (H3N2)), and Newcastle disease virus (NDV), were propagated in the allantoic cavity of 11-day-old embryonated chicken eggs. Each virus stock $(100 \mu \mathrm{L})$ was inoculated into the allantoic cavities of chicken eggs. After 72 -h incubation at $37^{\circ} \mathrm{C}$, eggs were chilled overnight at $4{ }^{\circ} \mathrm{C}$. The allantoic fluid containing the propagated viruses was harvested and purified by centrifugation at 4,000 rpm for $20 \mathrm{~min}$. The supernatant was frozen at $-80^{\circ} \mathrm{C}$ for long-term storage and direct use in the assay. Porcine epidemic diarrhea virus (PEDV) and canine distemper virus (CDV) were propagated in Vero cells, and porcine reproductive and respiratory syndrome (PRRS) and infectious canine hepatitis virus (ICHV) were propagated in MARC-145 and Madin-Darby canine kidney (MDCK) cell lines respectively. After 72 -h incubation at $37^{\circ} \mathrm{C}$, the cells were chilled overnight at $4{ }^{\circ} \mathrm{C}$. The fluid containing the propagated viruses was harvested and purified by centrifugation at 4,000 rpm for $20 \mathrm{~min}$. The supernatant was frozen at $-80{ }^{\circ} \mathrm{C}$ for long-term storage and direct use in the assay. In turn, differents typse of virus were prepared such as NDV $\left(10^{6} \mathrm{EID}_{50} / \mathrm{mL}\right)$, H1N1 $\left(10^{55} \mathrm{TCID}_{50} / \mathrm{mL}\right)$, H3N2 $\left(10^{5.25} \mathrm{TCID}_{50} / \mathrm{mL}\right)$, PRRS $\left(10^{6.5} \mathrm{TCID}_{50} / \mathrm{mL}\right)$, ICHV $\left(10^{6}\right.$ 
$\left.\mathrm{TCID}_{50} / \mathrm{mL}\right), \quad$ PEDV $\left(10^{6} \quad \mathrm{TCID}_{50} / \mathrm{mL}\right)$, and $\mathrm{CDV}\left(10^{4}\right.$ $\left.\mathrm{TCID}_{50} / \mathrm{mL}\right)$.

\subsection{Virus detection method}

The virus detection procedure was conducted by simply adding the viral strains in various viral titer $(50 \mu \mathrm{L})$ into $50 \mu \mathrm{L}$ PCPVs at a concentration of $0.0625 \mathrm{mg} \cdot \mathrm{mL}^{-1}$ in a 96 -well plate. The mixture was incubated for $15 \mathrm{~min}$ at room temperature, and the optical response of the PCPVs against the viral strain was evaluated.

\section{Results and discussion}

\subsection{Fabrication and characterization of CPV}

To fabricate polyaniline and polypyrrole encapsulated vesicles, block copolymers mPEG-b-pPhe and CM-PEG-b-pPhe were synthesized via ring-opening polymerization processes. Polymerization was confirmed using ${ }^{1} \mathrm{H}$ NMR and FT-IR. Polymeric vesicles consisting of the block copolymers were prepared by the emulsion solvent evaporation method and by loading aniline and pyrrole on the vesicles by simple mixing. Aniline and pyrrole loaded in the polymeric vesicles were oxidized with ammonium persulfate, while the absorbance of the particles was measured by time interval. One of controllable factors in synthesis of the systems based on conductive polymers is polymerization time, determining electrical or optical property responding to the stimulation [44]. By increasing polymerization time, the chain length of conductive polymer is changed, showing different spectra to physicochemical environment. After $24 \mathrm{~h}$ of oxidation, the characteristic absorbance spectra of CPVs were indistinguishable through $\mathrm{pH}$ variation (Fig. 2(a)). CPV polymerized for $48 \mathrm{~h}$ shows distinctive absorbance spectrum responding to various $\mathrm{pH}$ conditions, signaling an optical response to the impetus. Further investigation of polymerization time of CPV in longer period exposes insignificant difference from $48 \mathrm{~h}$ (Fig. S4 in the ESM). Ideally, the oxidation process should be conducted for at least $48 \mathrm{~h}$ to retain an optical property, enabling a sufficient optical response to the impetus (Fig. 2(b)). The morphology of CPVs was analyzed by dynamic light scattering (DLS) (Fig. 2(c)) and transmission electron microscopy (TEM) (Fig. 2(d)). The hydrodynamic diameter of the polymeric vesicles was $218 \mathrm{~nm}$ and maintained a spherical shape during oxidation of the conductive polymers. The oxidation time of the conductive polymers in the vesicles was determined to be $48 \mathrm{~h}$ for the CPVs to become available to transduce exterior stimulations to optical signals and maintain a stable structure. The stable nanostructure of CPV was explained by the contribution of the interaction between the aromatic rings belonging to polyaniline, polypyrrole, and polyphenyl in mPEG-b-pPhe, in accordance with a previous report [45]. Furthermore, CPV was stable in aqueous solution because amphiphilic block copolymers had enclosed the highly hydrophobic polyaniline and polypyrrole.

\subsection{Analysis of optical response of CPV to mechanical stimulus}

One of the characteristics of conductive polymers is their unique optical properties that are caused by electron distribution. CPV consisting of PANI has a distinctive absorption range because it absorbs the photon energy that matches the energy gap between the low energy level and the high energy level $[46,47]$. This energy gap could be shifted by intermolecular interactions, such as steric effects, when the distance of each molecule is sufficiently close $[48,49]$. Steric effects in response to the distance between particles can be demonstrated by the double layer force equation describing the repulsive interaction energy $W(D)_{\mathrm{R}}$ due to electric
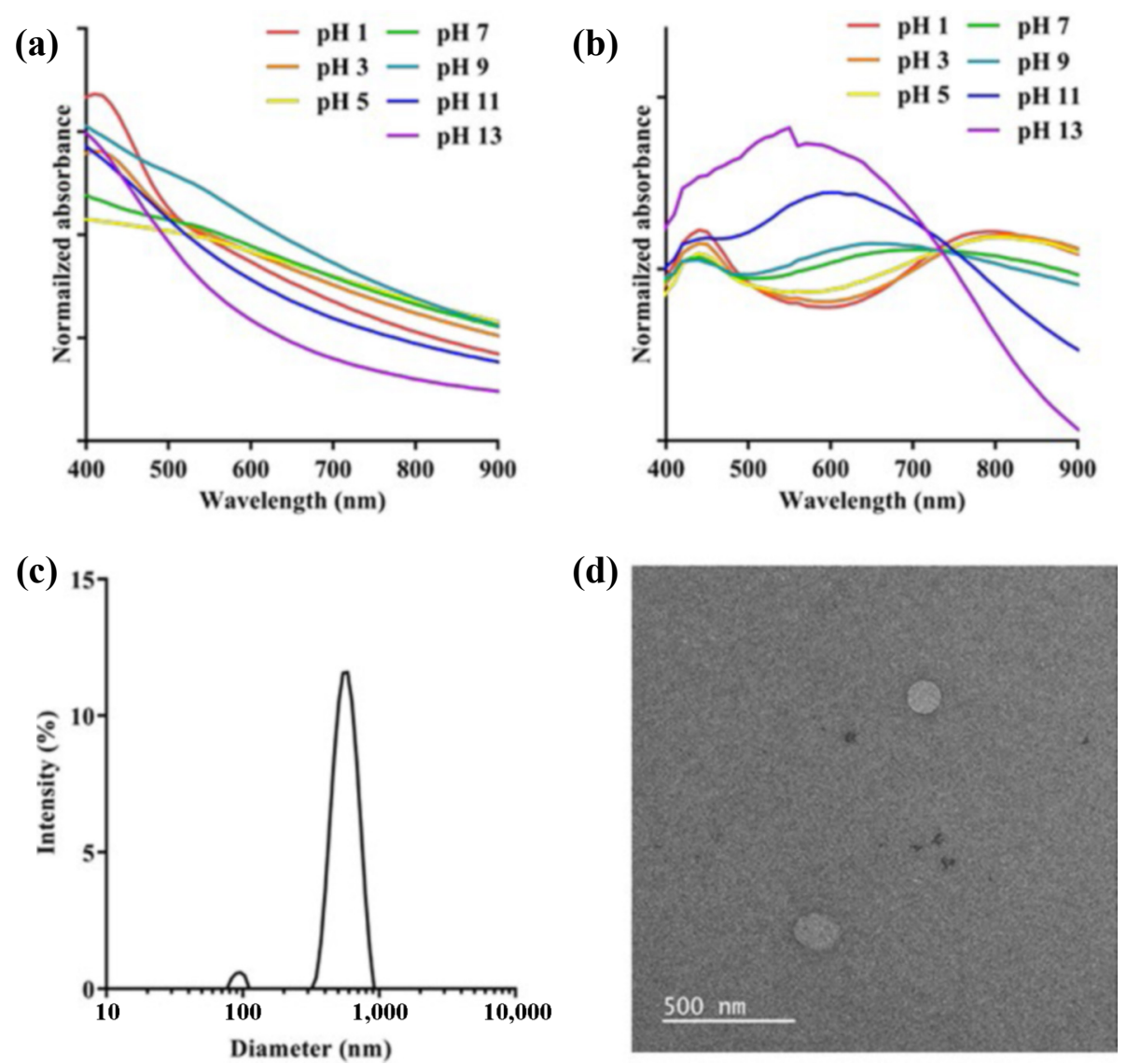

Figure 2 Ultraviolet-visible (UV-vis) absorbance spectrum of CPV, wherein polyaniline and polypyrrole were polymerized for (a) $24 \mathrm{~h}$ and (b) $48 \mathrm{~h}$. Absorbance spectrum was measured in $\mathrm{pH}$ range from 1 to 13. Morphology of CPV was analyzed by (c) DLC (d) TEM. 
repulsion [50]

$$
W(D)_{\mathrm{R}}=\frac{64 \pi k_{\mathrm{B}} T R \rho_{\infty} \gamma^{2}}{k^{2}} e^{-k D}
$$

Where $k_{\mathrm{B}}$ is the boltzmann constant, $R$ is the radius of particle, $\rho_{\infty}$ is the number density of ion in the bulk solution, $\gamma$ is the surface tension, $k$ is the Debye screening length, and $D$ is the distance between two spherical particles. From Eq. (2), we can see that the electric repulsion has an exponential decay correlation with the distance between the particles. Therefore, in the case of CPV aggregation, the distance between particles could be regarded as a mechanical factor for inducing a shift in their energy gap, involving a transition of the absorption spectrum. As a tool for verifying a mechanical stimulation that induces an optical response, we adopted a concentration of particles with a dependence on the mean distance in the disperse system [51].

Absorbance spectra of CPVs at various concentration were measured to confirm the optical response to the mechanical stimulus, i.e., the distance between CPVs (Fig.3(a)). As the concentration of CPVs increased, the absorption intensity of CPVs near $430 \mathrm{~nm}$ increased, while the absorption intensity near $630 \mathrm{~nm}$ decreased. The transition of the absorbance spectrum could be explained by the increase in intermolecular interaction between CPVs at a short distance, which elevates the transition of the energy state of CPVs. When CPVs were held together using a linker, hexamethylenediamine, the absorbance spectrum showed a relatively weak shift upon varying the concentration of CPVs from lower to higher (Fig. 3(b)). The result exposed the suppression of the absorbance spectrum shift by limiting the distance change between CPV, especially at lower concentrations. Intermolecular interaction between CPV particles was highly affected by the distance between CPVs, and this was elucidated by the transition of the absorbance spectrum; therefore, mechanical stimulus could be introduced by optical signals (Fig. 3(c)).
To analyze the shift of absorbance spectrum of CPVs by the mechanical stimulus, a new calculation method based on absorption intensity was used to transduce the variance into quantitative data, and the calculated value, optical response, was plotted against concentration (Fig. 3(d)). The optical response of CPVs increased as the mean distance between CPVs increased by 2-fold serial dilution from an initial concentration of 0.25 $\mathrm{mg} \cdot \mathrm{mL}^{-1}$. When the CPVs were forced to bind to each other via conjugation with a linker (CPV-L), the optical response induced by the same serial dilution was restricted compared to CPV without linkage, especially in the lower concentration range. The difference between the optical response of CPV and CPV-L was maximal at $0.03125 \mathrm{mg} \cdot \mathrm{mL}^{-1}$. The broader size distribution of CPV-L compared to CPV can be explained by the linkage between CPVs by the hexamethylenediamine-induced stack of the nanoparticles in CPV-L (Fig. 2(e)).

To optimize the optical response of CPV, triggered by the binding effect of influenza virus, the ratio of the surface functional group was assessed. We prepared various CPVs with different percentages of functional groups $(20 \%, 50 \%, 80 \%$, and $100 \%)$ by mixing block copolymers mPEG-b-pPhe and CM-PEG-b-pPhe. The optical response of CPV and CPV-L at different percentages of functional groups was presented (Fig. S5 in the ESM). When the percentage of the function group is higher, the difference in optical response between linked and non-linked CPVs is larger (Fig. 4(a)). The optimal percentage of functional groups in CPVs is $80 \%$, exposing maximal optical response to binding effect (Fig. 4(b)), and the optimal concentration exhibiting the highest ratio of optical responses of CPV and CPV-L was 0.03125 $\mathrm{mg} \cdot \mathrm{mL}^{-1}$ (Fig. 4(c)). Optimal hydrophilic and hydrophobic ratio (fvalue) of $\mathrm{CPV}-\mathrm{L}$ was evaluted at $0.36,0.49$, and 0.58 and determined to 0.49 exposing the most restricted optical response by linkage (Fig. S5(a) in the ESM). According to a coherent experimental data, it could be said that CPVs have optical (a)

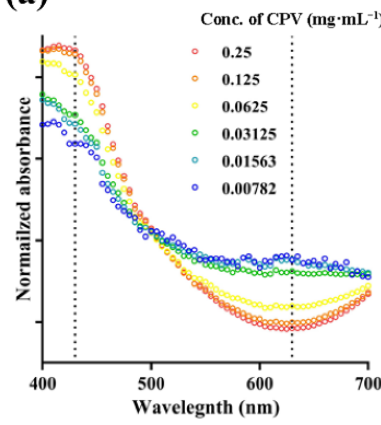

(d)

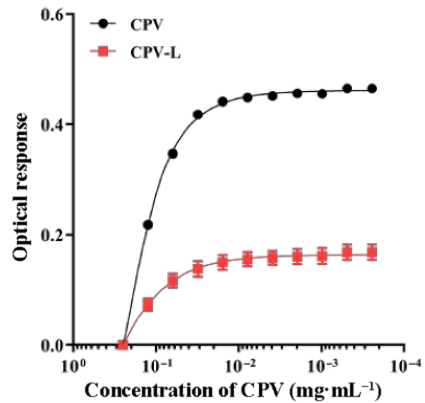

(b)

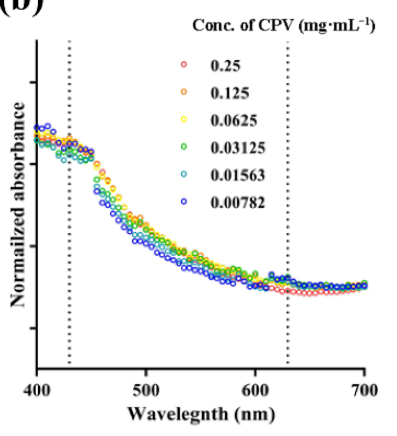

(e)

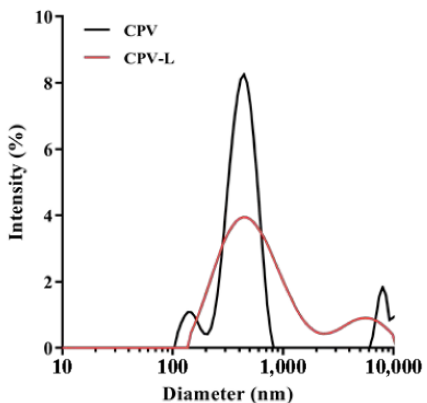

(c)

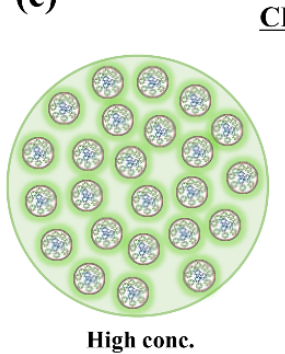

CPV

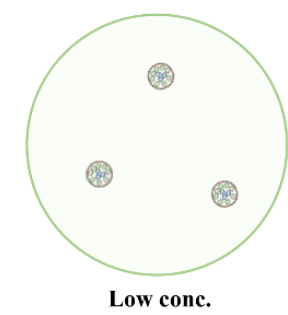

Change of distance between CPVs $\rightarrow$ Optical response

CPV-L

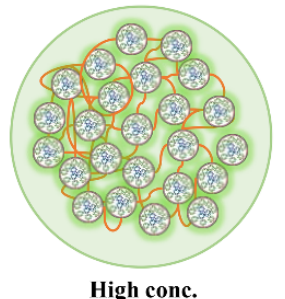

High conc.

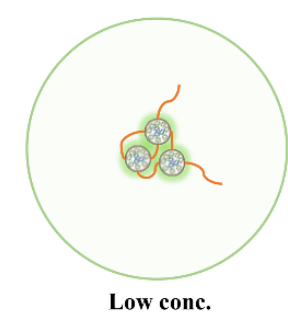

Low conc.
Restricted change of distance between CPVs $\rightarrow$ Weak optical response

Figure 3 Characterization of the mechanism of response of CPVs to mechanical stimuli and the transduction into optical signals. UV-vis absorbance spectrum of (a) CPV and (b) CPV-L, a CPV conjugated by a linker (hexamethylenediamine). Serial dilution (2-fold) was performed to measure UV-vis absorbance. (c) Schematic representation of intermolecular interaction of CPV determined by the distance between nanoparticles. Quantification of absorbance change at 430 and 630 nm was calculated using the formula for optical response and fitted with (d) sigmoidal curve. (e) Size distributions of CPV and CPV-L were measured by DLS. Data represent mean \pm standard deviation $(n=3)$. 

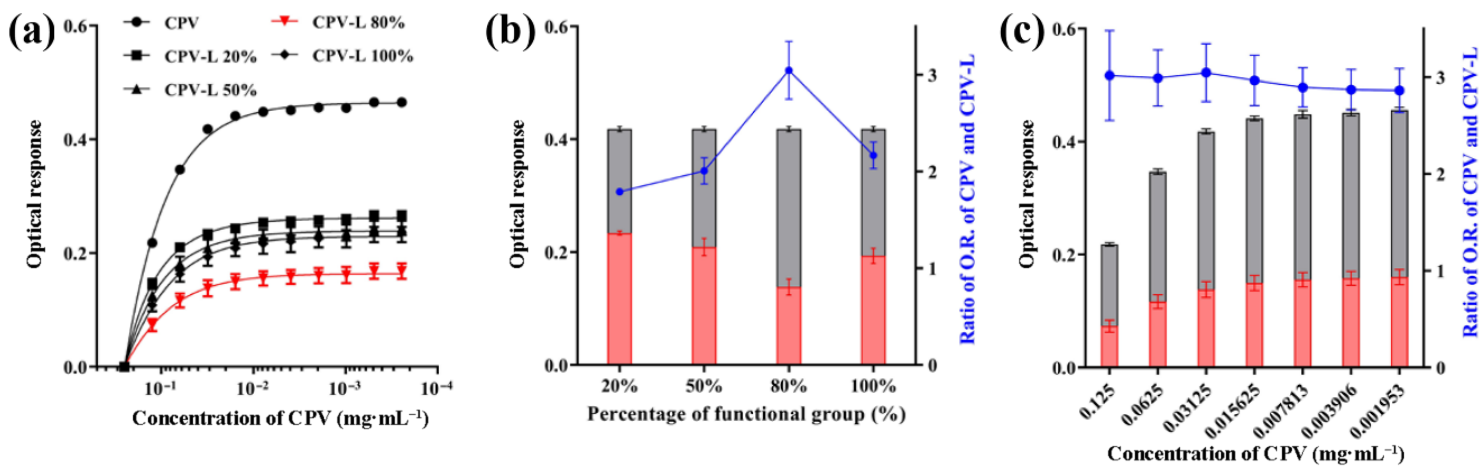

Figure 4 (a) Optical responses of CPV and CPV-L synthesized with CM-PEG-pPhe containing different percentages functional groups (20\%, 50\%, 80\%, and 100\%) were calculated in various concentrations diluted by 2-fold and fitted to a sigmoidal curve. (b) Optical responses of CPV and CPV-L synthesized with CM-PEG-pPhe containing different percentages of functional groups are compared. (c) The ratio of optical response of CPV and CPV-L containing 80\% of CM-PEG-b-pPhe has been presented for various concentrations. Data represent mean \pm standard deviation $(n=3)$.

response to mechanical stimulus but also, the degree of response is controllable. The aggregation phenomena of CPV by linker were further analyzed by scanning electron microscopy (SEM). The image of CPV-L exposes linked morphology whereas measured image of CPV shows individually distributed particles with sizes around $200 \mathrm{~nm}$. (Fig. S6 in the ESM).

\subsection{H1N1 virus detection by PCPV}

PCPV was prepared by conjugating CPV and peptide specifically binding to $\mathrm{H} 1 \mathrm{~N} 1$ virus. PCPV $\left(0.0625 \mathrm{mg} \cdot \mathrm{mL}^{-1}\right)$ was mixed with the same volume of $\mathrm{H} 1 \mathrm{~N} 1$ virus stocks in different viral titers and incubated for $15 \mathrm{~min}$ at room temperature. Specific binding of influenza virus with peptide tethered on the surface of PCPV induced agglomeration of the bound complexes, which was a mechanical stimulation eliciting a shift of $\pi-\pi$ interactions between CPVs [52]. Absorbance spectra were measured using UV-vis spectroscopy. As shown in Fig. 5(a), the response of PCPV to the stimulus corresponded to a shift in the absorbance spectrum, increasing the absorbance intensity at approximately 430 and decreasing absorbance intensity at approximately $630 \mathrm{~nm}$. In the case of PCPV binding to the virus, the distance between particles was close enough to enable $\pi-\pi$ interactions in the conductive polymers [53-55]. The binding event of PCPV and H1N1 was visualized by TEM (Fig. S7 in the ESM). On the other hand, CPV showed scarce absorbance change when mixed with H1N1 virus (Fig. 5(b)). Considering PCPV showed a lack of difference in particle characterization result with $\mathrm{CPV}$, the existence of peptides on the surface of the PCPV is essential for the selective binding of PCPV and virus (Fig. S7(a) in the ESM). To analyze the degree of response of PCPV to the target virus, in terms of absorbance, the optical response was calculated by adopting an inventive calculation method combining the relative absorption change at $430 \mathrm{~nm}$ and $630 \mathrm{~nm}$, with respect to the blank (Fig. 5(c)). The optical response of PCPV against H1N1 showed robust linear dependence, indicating that the designed biosensor successfully transduced the mechanical stimulation to the optical response for rapid quantitative detection of the target virus. The correlation between the measured optical response of PCPV and viral titer was evaluated, and the results showed a broad dynamic range from 5.20 to $3.39 \log _{10} \mathrm{TCID}_{50} / \mathrm{mL}$ of H1N1 with a correlation coefficient $\left(R^{2}\right)$ of 0.9493 . The limit of detection was determined to be $3.37 \log _{10} \mathrm{TCID}_{50} / \mathrm{mL}$, proving the feasibility of the designed platform as a biosensor for $\mathrm{H} 1 \mathrm{~N} 1$ virus. Optical $f$ value of PCPV was evaluated at 0.36, 0.49, and 0.58 and determined to 0.49 exposing the maximal optical response toward $\mathrm{H} 1 \mathrm{~N} 1$ virus (Fig. S8(b) in the ESM).

\subsection{Cross-reactivity test of PCPV}

Specificity is an essential element for diagnostic assays because generation of false-negative results can decrease the reliability of the assay, despite having an ultra-low sensitivity [56]. To confirm the specificity of the designed system, we conducted crossreactivity test of PCPV against the target $\mathrm{H} 1 \mathrm{~N} 1$ virus and different species of viruses such as NDV, ICHV, PEDV, PRRS, CDV, and other subtype of influenza virus, H3N2. Absorbance peak of cellculture media was observed at 560nm in ICHV, PEDV, PPRS, and CDV but, making a limited impact on the analysis of optical response of PCPV against testing viruses.

The absorbance spectrum of PCPV incubated with non-target viruses showed negligible transition, and the optical response of the system exhibited no quantitative signal transduction, in contrast with the target virus $\mathrm{H} 1 \mathrm{~N} 1$, which elicited a distinctive signal transduction (Fig. 6). The designed diagnostic platform with specific molecular recognition successfully identified the H1N1 virus, enabling rapid and selective detection of the target virus.
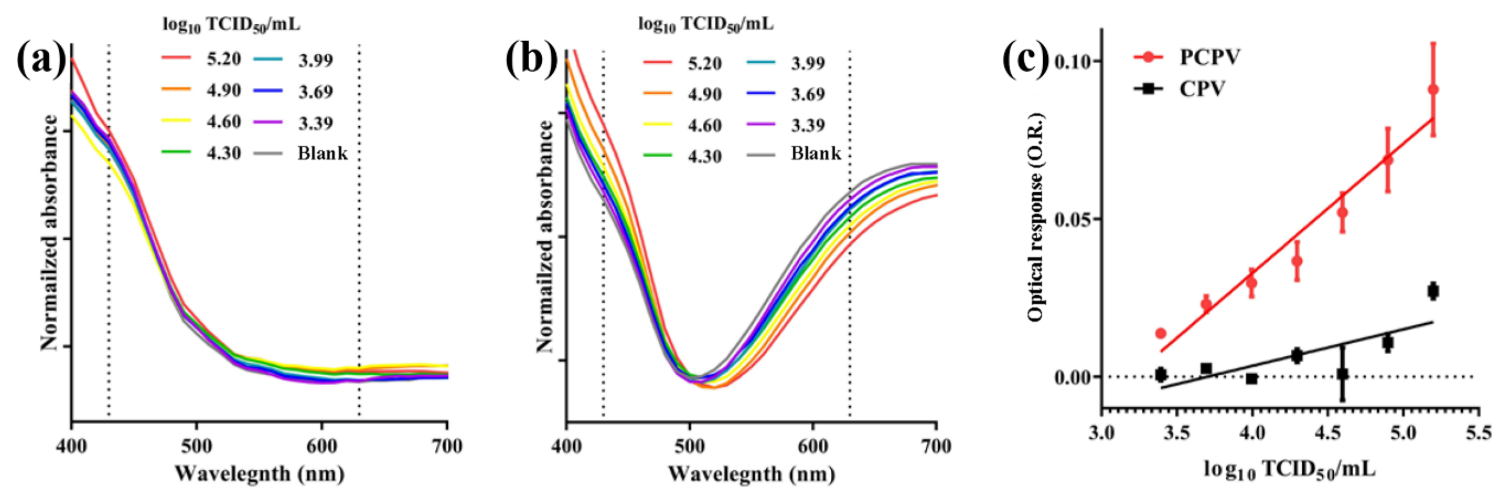

Figure 5 UV-vis absorbance spectra of (a) CPV and (b) CPV conjugated with peptide (PCPV), after the incubation, are shown. (c) Optical response of CPV and PCPV toward CA04 (H1N1 virus) were calculated and fitted. Data represent mean $\pm \operatorname{standard~deviation~}(n=3)$. 
(a)

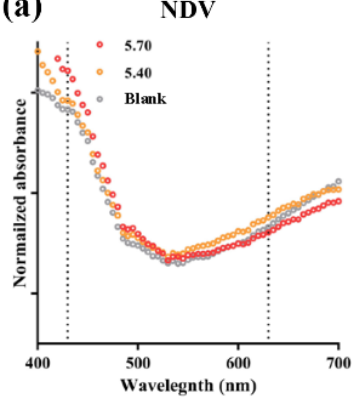

(e)

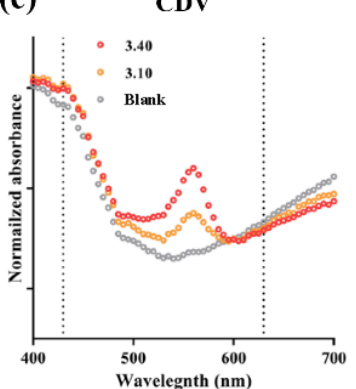

(b)

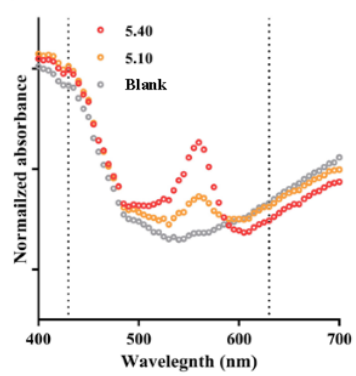

(f)

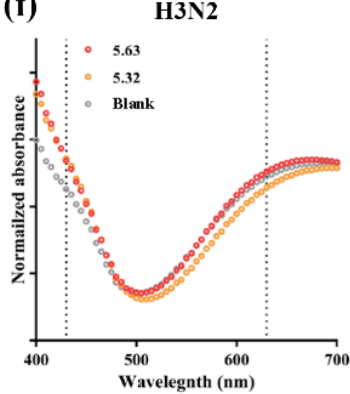

(c)

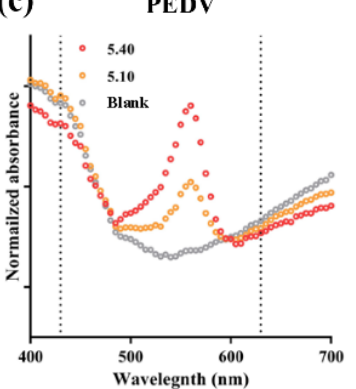

(g)

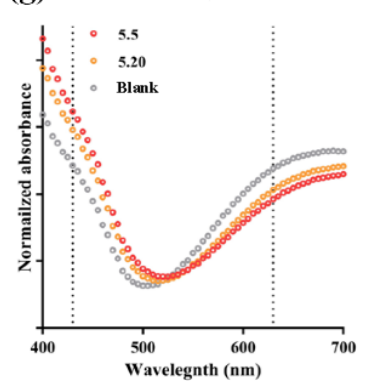

(d)

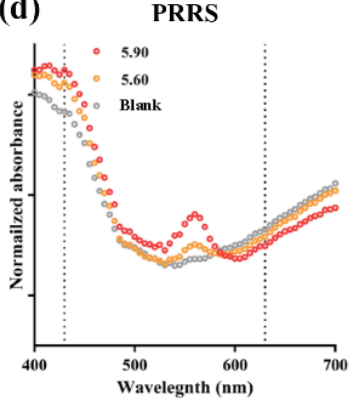

(h)

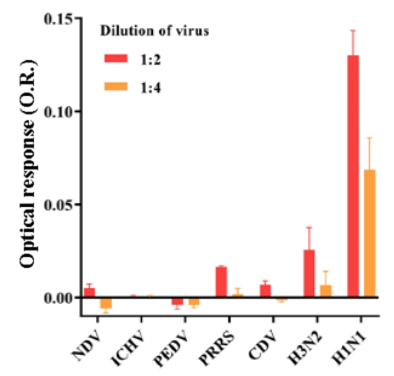

Figure 6 Specificity of PCPV toward H1N1 was evaluated. UV-vis absorbance spectrum of PCPV incubated with (a) NDV, (b) ICHV, (c) PEDV, (d) PRRS, (e) $\mathrm{CDV}$, (f) H3N2, and (g) H1N1 was measured. Viral titer was presented in (a) $\log _{10} \mathrm{EID}_{50} / \mathrm{mL}$ and (b)-(g) $\log _{10} \mathrm{TCID}_{50} / \mathrm{mL}$. Absorbance peak of cell-culture media was observed at $560 \mathrm{~nm}$ in ICHV, PEDV, PPRS, and CDV. (h) Optical response of PCPV to virus specimen was calculated and fitted. Absorbance of PCPV had no distinctive change to non-target viruses. Data represent mean \pm standard deviation $(n=3)$.

\section{Conclusions}

Herein, we developed a rapid detection platform for specifically transducing the binding of the target virus into an optical response by adopting polymeric vesicles comprising conductive polymers. The unique property of the system to generate an optical response by mechanical stimulation was carefully evaluated. This detection system elicited a quantitative optical response against various titers of H1N1 in $15 \mathrm{~min}$, demonstrating the advantage of the platform in enabling a rapid and quantitative detection of the target virus. We achieved a limit of detection of $10^{337} \mathrm{TCID}_{50} / \mathrm{mL}$ and showed a wide dynamic range for the second power of ten in the $\mathrm{TCID}_{50} / \mathrm{mL}$. The designed system not only possesses a sufficient level of limit of detection and dynamic range but also targetresponsive signal transduction, establishing specificity by employing a peptide having a molecular recognition to hemagglutinin. Consequently, this platform was demonstrated to meet the fundamental aspects of diagnostics. In addition, the simple process of detection provides less burden to operators because of reduced requirements, such as skilled operation techniques and additional equipment. Thus, we believe that the designed system can be used as a complementary platform for the rapid detection of the target viruses, and further expanding its application will contribute to the prompt execution of preventative measures against the spread of viral infections.

\section{Acknowledgements}

H.-O. Kim acknowledges support from the National Research Foundation of Korea grant funded by the Korean government (No. NRF-2019R1I1A1A01057005) and Evaluation for Technology in Food, Agriculture and Forestry (IPET) through the Animal Disease Management Technology Development Program funded by Ministry of Agriculture, Food and Rural Affairs (MAFRA) (No. 320056-2). D. Song acknowledges support from Korea Mouse Phenotyping Project (No. NRF2019M3A9D5A01102797) and Development of African Swine Fever Virus Vaccine and Assessment of Rapid Test Kit (No. NRF2019K1A3A1A61091813) of the Ministry of Science and ICT through the National Research Foundation. S. Haam acknowledges support from Technology Development Project for Biological Hazards Management in Indoor Air Program of Korea Environment Industry \& Technology Institute (KEITI) funded by Korea Ministry of Environment (MOE) (No. RE202101004) and Nano Material Technology Development Program through the National Research Foundation of Korea (NRF) funded by the Ministry of Education, Science and Technology (No. 2017M3A7B4041798). This research was also supported by the Bio \& Medical Technology Development Program (No. NRF2018M3A9E2022819) and the Bio \& Medical Technology Development Program (No. NRF-2018M3A9H4056340) of the National Research Foundation (NRF) funded by the Ministry of Science \& ICT.

Electronic Supplementary Material: Supplementary material (Table S1 and Figs. S1-S8) is available in the online version of this article at https://doi.org/10.1007/s12274-021-3772-6.

\section{References}

[1] Smith, G. J. D.; Vijaykrishna, D.; Bahl, J.; Lycett, S. J.; Worobey, M.; Pybus, O. G.; Ma, S. K.; Cheung, C. L.; Raghwani, J.; Bhatt, S. et al. Origins and evolutionary genomics of the 2009 swine-origin H1N1 influenza A epidemic. Nature 2009, 459, 1122-1125.

[2] Vijaykrishna, D.; Smith, G. J. D.; Pybus, O. G.; Zhu, H. C.; Bhatt, S.; Poon, L. L. M.; Riley, S.; Bahl, J.; Ma, S. K.; Cheung, C. L. et al. Long-term evolution and transmission dynamics of swine influenza A virus. Nature 2011, 473, 519-522.

[3] Wonderlich, E. R.; Swan, Z. D.; Bissel, S. J.; Hartman, A. L.; Carney, J. P.; O'Malley, K. J.; Obadan, A. O.; Santos, J.; Walker, R.; Sturgeon, T. J. et al. Widespread virus replication in alveoli drives acute respiratory distress syndrome in aerosolized H5N1 influenza infection of macaques. J. Immunol. 2017, 198, 1616-1626.

[4] Grubaugh, N. D.; Ladner, J. T.; Lemey, P.; Pybus, O. G.; Rambaut, A.; Holmes, E. C.; Andersen, K. G. Tracking virus outbreaks in the twenty-first century. Nat. Microbiol. 2019, 4, 10-19.

[5] Parvez, M. K.; Parveen, S. Evolution and emergence of pathogenic viruses: Past, present, and future. Intervirology 2017, 60, 1-7.

[6] Ke, C. W.; Mok, C. K. P.; Zhu, W. F.; Zhou, H. B.; He, J. F.; Guan, 
W. D.; Wu, J.; Song, W. J.; Wang, D. Y.; Liu, J. X. et al. Human infection with highly pathogenic avian influenza $\mathrm{A}(\mathrm{H} 7 \mathrm{~N} 9)$ virus, China. Emerg. Infect. Dis. 2017, 23, 1332-1340.

[7] Tang, J.; Zhang, J.; Zhou, J. F.; Zhu, W. F.; Yang, L.; Zou, S. M.; Wei, H. J.; Xin, L.; Huang, W. J.; Li, X. Y. et al. Highly pathogenic avian influenza H7N9 viruses with reduced susceptibility to neuraminidase inhibitors showed comparable replication capacity to their sensitive counterparts. Virol. J. 2019, 16, 87.

[8] Kosack, C. S.; Page, A. L.; Klatser, P. R. A guide to aid the selection of diagnostic tests. Bull. World Health Organ. 2017, 95, 639-645.

[9] Land, K. J.; Boeras, D. I.; Chen, X. S.; Ramsay, A. R.; Peeling, R. W. REASSURED diagnostics to inform disease control strategies, strengthen health systems and improve patient outcomes. Nat. Microbiol. 2019, 4, 46-54.

[10] Mahony, J. B.; Petrich, A.; Smieja, M. Molecular diagnosis of respiratory virus infections. Crit. Rev. Clin. Lab. Sci. 2011, 48, 217-249.

[11] Brittain-Long, R.; Andersson, L. M.; Olofsson, S.; Lindh, M.; Westin, J. Seasonal variations of 15 respiratory agents illustrated by the application of a multiplex polymerase chain reaction assay. Scand. J. Infect. Dis. 2012, 44, 9-17.

[12] Kang, X. P.; Li, Y. C.; Fan, L.; Lin, F.; Wei, J. J.; Zhu, X. L.; Hu, Y.; Li, J.; Chang, G. H.; Zhu, Q. Y. et al. Development of an ELISAarray for simultaneous detection of five encephalitis viruses. Virol. $J$. 2012, 9, 56.

[13] Cho, C. H.; Woo, M. K.; Kim, J. Y.; Cheong, S.; Lee, C. K.; An, S. A.; Lim, C. S.; Kim, W. J. Evaluation of five rapid diagnostic kits for influenza A/B virus. J. Virol. Methods 2013, 187, 51-56.

[14] Boonham, N.; Kreuze, J.; Winter, S.; van der Vlugt, R.; Bergervoet, J.; Tomlinson, J.; Mumford, R. Methods in virus diagnostics: From ELISA to next generation sequencing. Virus Res. 2014, 186, 20-31.

[15] Krammer, F.; Smith, G. J. D.; Fouchier, R. A. M.; Peiris, M.; Kedzierska, K.; Doherty, P. C.; Palese, P.; Shaw, M. L.; Treanor, J.; Webster, R. G. et al. Influenza. Nat. Rev. Dis. Primers 2018, 4, 3.

[16] Zumla, A.; Al-Tawfiq, J. A.; Enne, V. I.; Kidd, M.; Drosten, C.; Breuer, J.; Muller, M. A.; Hui, D.; Maeurer, M.; Bates, M. et al. Rapid point of care diagnostic tests for viral and bacterial respiratory tract infections - needs, advances, and future prospects. Lancet Infect. Dis. 2014, 14, 1123-1135.

[17] Chan, W. C. W. Nano research for covid-19. ACS Nano 2020, 14, 3719-3720.

[18] Bang, D.; Chang, Y. W.; Park, J.; Lee, J.; Yoo, K. H.; Huh, Y. M.; Haam, S. Fabrication of a near-infrared sensor using a polyaniline conducting polymer thin film. Thin Solid Films 2012, 520, 6818-6821.

[19] Choi, J.; Hong, Y.; Lee, E.; Kim, M. H.; Yoon, D. S.; Suh, J.; Huh, Y.; Haam, S.; Yang, J. Redox-sensitive colorimetric polyaniline nanoprobes synthesized by a solvent-shift process. Nano Res. 2013, 6, 356-364.

[20] Balint, R.; Cassidy, N. J.; Cartmell, S. H. Conductive polymers: Towards a smart biomaterial for tissue engineering. Acta Biomater. 2014, 10, 2341-2353.

[21] Lee, T.; Bang, D.; Park, Y.; Chang, Y. W.; Kang, B.; Kim, J.; Suh, J. S.; Huh, Y. M.; Haam, S. Synthesis of stable magnetic polyaniline nanohybrids with pyrene as a cross-linker for simultaneous diagnosis by magnetic resonance imaging and photothermal therapy. Eur. J. Inorg. Chem. 2015, 2015, 3740-3747.

[22] Uram, J. D.; Ke, K.; Hunt, A. J.; Mayer, M. Submicrometer porebased characterization and quantification of antibody-virus interactions. Small 2006, 2, 967-972.

[23] Veetil, J. V.; Ye, K. Development of immunosensors using carbon nanotubes. Biotechnol. Prog. 2007, 23, 517-531.

[24] Silva, M. M. S.; Dias, A. C. M. S.; Silva, B. V. M.; Gomes-Filho, S. L. R.; Kubota, L. T.; Goulart, M. O. F.; Dutra, R. F. Electrochemical detection of dengue virus NS1 protein with a poly(allylamine)/carbon nanotube layered immunoelectrode. $J$. Chem. Technol. Biotechnol. 2015, 90, 194-200.

[25] Min, H. G.; Kim, J. G.; Jang, G. S.; O, E. J. Effect of surfactant on the properties of tantalum capacitor using soluble polyaniline electrolyte. Korean Chem. Eng. Res. 2003, 41, 343. http://www.koreascience.or.kr/article/JAKO200310103436338.page
[26] Sheridan, E. M.; Breslin, C. B. Enantioselective detection of D- and 1phenylalanine using optically active polyaniline. Electroanalysis 2005, 17, 532-537.

[27] Tran, L. D.; Nguyen, D. T.; Nguyen, B. H.; Do, Q. P.; Nguyen, H. L. Development of interdigitated arrays coated with functional polyaniline/MWCNT for electrochemical biodetection: Application for human papilloma virus. Talanta 2011, 85, 1560-1565.

[28] Bang, D.; Lee, J.; Park, J.; Choi, J.; Chang, Y. W.; Yoo, K. H.; Huh Y. M.; Haam, S. Effectively enhanced sensitivity of a polyaniline-carbon nanotube composite thin film bolometric nearinfrared sensor. J. Mater. Chem. 2012, 22, 3215-3219.

[29] Choi, E. B.; Choi, J.; Bae, S. R.; Kim, H. O.; Jang, E.; Kang, B.; Kim, M. H.; Kim, B.; Suh, J. S.; Lee, K. et al. Colourimetric redoxpolyaniline nanoindicator for in situ vesicular trafficking of intracellular transport. Nano Res. 2015, 8, 1169-1179.

[30] Shrivastav, A. M.; Usha, S. P.; Gupta, B. D. A localized and propagating spr, and molecular imprinting based fiber-optic ascorbic acid sensor using an in situ polymerized polyaniline-Ag nanocomposite. Nanotechnology 2016, 27, 345501.

[31] Takemura, K.; Adegoke, O.; Takahashi, N.; Kato, T.; Li, T. C.; Kitamoto, N.; Tanaka, T.; Suzuki, T.; Park, E. Y. Versatility of a localized surface plasmon resonance-based gold nanoparticle-alloyed quantum dot nanobiosensor for immunofluorescence detection of viruses. Biosens. Bioelectron. 2017, 89, 998-1005.

[32] Botewad, S. N.; Pahurkar, V. G.; Muley, G. G. Fabrication and evaluation of evanescent wave absorption based polyanilinecladding modified fiber optic urea biosensor. Opt. Fiber Technol. 2018, 40, 8-12.

[33] Ramos, K. C.; Nishiyama, K.; Maeki, M.; Ishida, A.; Tani, H.; Kasama, T.; Baba, Y.; Tokeshi, M. Rapid, sensitive, and selective detection of $\mathrm{H} 5$ hemagglutinin from avian influenza virus using an immunowall device. ACS Omega 2019, 4, 16683-16688.

[34] Maddali, H.; Miles, C. E.; Kohn, J.; O'Carroll, D. M. Optical biosensors for virus detection: Prospects for SARS-CoV-2/COVID19. ChemBioChem 2021, 22, 1176-1189.

[35] Cho, S.; Lee, J. S.; Joo, H. Recent developments of the solutionprocessable and highly conductive polyaniline composites for optical and electrochemical applications. Polymers (Basel) 2019, 11, 1965.

[36] Su, N. Improving electrical conductivity, thermal stability, and solubility of polyaniline-polypyrrole nanocomposite by doping with anionic spherical polyelectrolyte brushes. Nanoscale Res. Lett. 2015, 10,301

[37] Khrenova, M. G.; Nemukhin, A. V.; Tsirelson, V. G. Origin of the $\pi$ stacking induced shifts in absorption spectral bands of the green fluorescent protein chromophore. Chem. Phys. 2019, 522, 32-38

[38] Song, S.; Ha, K.; Guk, K.; Hwang, S. G.; Choi, J. M.; Kang, T.; Bae, P.; Jung, J.; Lim, E. K. Colorimetric detection of influenza A (H1N1) virus by a peptide-functionalized polydiacetylene (PEPPDA) nanosensor. $R S C A d v$. 2016, 6, 48566-48570.

[39] Salonen, L. M.; Ellermann, M.; Diederich, F. Aromatic rings in chemical and biological recognition: Energetics and structures. Angew. Chem., Int. Ed. 2011, 50, 4808-4842.

[40] Wu, S. Q.; Wang, J. W.; Shao, J.; Wei, L.; Yang, K.; Ren, H. Building a novel chemically modified polyaniline/thermally reduced graphene oxide hybrid through $\pi-\pi$ interaction for fabricating acrylic resin elastomer-based composites with enhanced dielectric property. ACS Appl. Mater. Interfaces 2017, 9, 28887-28901.

[41] Zhang, L.; Zhang, Z. L.; Lv, Y. Y.; Chen, X. Q.; Wu, Z.; He, Y. Y.; Zou, Y. H. Reduced graphene oxide aerogels with uniformly selfassembled polyaniline nanosheets for electromagnetic absorption. ACS Appl. Nano Mater. 2020, 3, 5978-5986.

[42] Chevaliez, S.; Poiteau, L.; Rosa, I.; Soulier, A.; Roudot-Thoraval, F.; Laperche, S.; Hézode, C.; Pawlotsky, J. M. Prospective assessment of rapid diagnostic tests for the detection of antibodies to hepatitis $\mathrm{C}$ virus, a tool for improving access to care. Clin. Microbiol. Infect. 2016, 22, 459.e1-459.e6

[43] Luo, R.; Fongwen, N.; Kelly-Cirino, C.; Harris, E.; Wilder-Smith, A.; Peeling, R. W. Rapid diagnostic tests for determining dengue serostatus: A systematic review and key informant interviews. Clin. Microbiol. Infect. 2019, 25, 659-666.

[44] Navarchian, A. H.; Hasanzadeh, Z.; Joulazadeh, M. Effect of 
polymerization conditions on reaction yield, conductivity, and ammonia sensing of polyaniline. Adv. Polym. Technol. 2013, 32, 21356.

[45] Kim, H. O.; Yeom, M.; Kim, J.; Kukreja, A.; Na, W.; Choi, J.; Kang, A.; Yun, D.; Lim, J. W.; Song, D. et al. Reactive oxygen speciesregulating polymersome as an antiviral agent against influenza virus. Small 2017, 13, 1700818.

[46] Li, J. F.; Zhang, R. Q. Strong orbital deformation due to $\mathrm{CH}-\pi$ interaction in the benzene-methane complex. Phys. Chem. Chem. Phys. 2015, 17, 29489-29491.

[47] Liu, Y. F.; Ding, Y.; Gou, H. L.; Huang, X.; Zhang, G. Y.; Zhang, Q.; Liu, Y. Z.; Meng, Z.; Xi, K.; Jia, X. D. Room temperature synthesis of $\mathrm{pH}$-switchable polyaniline quantum dots as a turn-on fluorescent probe for acidic biotarget labeling. Nanoscale 2018, 10, 6660-6670.

[48] Wang, M.; Jang, S. K.; Jang, W. J.; Kim, M.; Park, S. Y.; Kim, S. W.; Kahng, S. J.; Choi, J. Y.; Ruoff, R. S.; Song, Y. J. et al. A platform for large-scale graphene electronics-CVD growth of singlelayer graphene on CVD-grown hexagonal boron nitride. Adv. Mater. 2013, 25, 2746-2752.

[49] Yang, J.; Zhen, X.; Wang, B.; Gao, X. M.; Ren, Z. C.; Wang, J. Q.; Xie, Y. J.; Li, J. R.; Peng, Q.; Pu, K. Y. et al. The influence of the molecular packing on the room temperature phosphorescence of purely organic luminogens. Nat. Commun. 2018, 9, 840.

[50] Mati, I. K.; Cockroft, S. L. Molecular balances for quantifying non- covalent interactions. Chem. Soc. Rev. 2010, 39, 4195-4205.

[51] Kryuchkov, Y. N. Concentration dependence of the mean interparticle distance in disperse systems. Refract. Ind. Ceram. 2001, $42,390-392$.

[52] Gribkova, O. L.; Nekrasov, A. A.; Ivanov, V. F.; Kozarenko, O. A.; Posudievsky, O. Y.; Vannikov, A. V.; Koshechko, V. G.; Pokhodenko, V. D. Mechanochemical synthesis of polyaniline in the presence of polymeric sulfonic acids of different structure. Synth. Metals 2013, 180, 64-72.

[53] Fan, G. L.; Yan, D. P. Two-component orderly molecular hybrids of diphenylanthracene: Modulation of solid-state aggregation toward tunable photophysical properties and highly enhanced electrochemiluminescence. Adv. Opt. Mater. 2016, 4, 2139-2147.

[54] Chen, T.; Li, M. X.; Liu, J. Q. $\pi-\pi$ stacking interaction: A nondestructive and facile means in material engineering for bioapplications. Cryst. Growth Des. 2018, 18, 2765-2783.

[55] Stewart, K.; Limbu, S.; Nightingale, J.; Pagano, K.; Park, B.; Hong, S.; Lee, K.; Kwon, S.; Kim, J. S. Molecular understanding of a $\pi$ conjugated polymer/solid-state ionic liquid complex as a highly sensitive and selective gas sensor. J. Mater. Chem. C 2020, 8, 15268-15276.

[56] Kilic, T.; Weissleder, R.; Lee, H. Molecular and immunological diagnostic tests of covid-19: Current status and challenges. iScience 2020, 23, 101406. 